\title{
Simplified setup for high-resolution spectroscopy that uses ultrashort pulses
}

\author{
E. Gershgoren, R. A. Bartels, J. T. Fourkas, ${ }^{*}$ R. Tobey, M. M. Murnane, and H. C. Kapteyn \\ JILA, University of Colorado, and the National Institute of Standards and Technology, Boulder, Colorado 80309-0440
}

Received September 3, 2002

\begin{abstract}
Using a broadband femtosecond laser and a simple optical setup, we demonstrate narrow-bandwidthtunable excitation of vibrational modes in $\mathrm{CCl}_{4}$ and $\mathrm{CHBr}_{3}$ liquids. The resolution obtained is 80 times higher than the laser bandwidth. A pair of time-shifted, linearly chirped pulses is created by use of a high-order dispersion-compensated prism-interferometer setup. We use this pulse pair to selectively excite Ramanactive transitions. Our setup represents a significant simplification with improved resolution, of previous approaches to the use of ultrashort pulses for chemically selective spectroscopy. (C) 2003 Optical Society of America
\end{abstract}

OCIS codes: 190.0190, 320.0320, 300.0300, 190.4380.

Chemical sensing is a prominent application of lasers. Typically, a target molecular species is detected by means of characteristic resonances. However, these resonances span a large region of the spectrum, broader than can be addressed by any single tunable laser system. This difficulty makes coherent Raman spectroscopy techniques, such as coherent anti-Stokes Raman scattering (CARS) spectroscopy and microscopy, coherent Stokes Raman scattering spectroscopy, and Kerr spectroscopy, attractive. ${ }^{1-3}$ In these techniques, vibrational transitions with energies in the mid- to far-infrared can be probed simply and over a broad range with visible or near-infrared lasers. For example, a pair of tunable synchronized picosecond lasers can excite a specific vibrational mode in a molecule corresponding to the frequency difference between the two pulses. ${ }^{4}$ The vibrational excitation can be detected by light scattering by use of either frequency shifts or depolarization. This approach makes possible high-resolution, broadly tunable, mode-specific excitation and chemical sensing.

Femtosecond light pulses possess a broad spectral bandwidth, making it possible to obtain both colors required for exciting a Raman-active mode from a single pulse. A transform-limited pulse will excite all the Raman-active modes accessible by the pulse bandwidth, which also corresponds to all modes with a period longer than the pulse duration. In this case it can be difficult to distinguish the target chemical species. Thus femtosecond lasers have traditionally been considered undesirable for many types of spectroscopy.

However, selective Raman excitation is possible with short, ${ }^{5}$ shaped, ${ }^{6-9}$ or chirped $^{9-11}$ femtosecond pulses that appropriately adjust the relative phase between different frequency components in a single pulse. Recently a femtosecond laser oscillator was used to implement chemically selective CARS microscopy with only a single laser beam by use of a liquid-crystal pulse shaper that imprinted a sinusoidally varying phase on the pulse. ${ }^{12}$

0146-9592/03/050361-03\$15.00/0
In this Letter we demonstrate a complementary technique that uses a femtosecond oscillator combined with simple optics and that achieves even higher resolution. In our approach, a femtosecond pulse is linearly chirped and split into two pulses. These pulses are subsequently recombined with a relative time delay. At any point in time the composite light field consists of a pair of instantaneous frequencies. If the pulses are linearly chirped, the difference between these two frequencies depends on the time delay and will not change with time even though the frequencies themselves are changing. The beating of these two fields creates a spectrum with regular modulations; as the pulse is strongly chirped, these regular modulations translate in the time domain into a periodic beat in the pulse intensity. The periodicity of this pulse train can be matched to the period of a single vibrational mode, permitting selective mode excitation with a simple adjustment of the relative delay between the chirped pump pulses. This approach is distinct from previous ones ${ }^{12}$ in that the pulse train is generated with spectral amplitude shaping as opposed to phase manipulation. The key to our advance was the design of a simple optical configuration that is capable of generating a pulse pair with a highly linear chirp. The result is a simple, low cost, higher-resolution optical system that can be used for chemical sensing of a broad range of molecules by use of only a single broadband laser oscillator.

Our experimental setup, shown in Fig. 1, consists of a 500-mW broadband Ti:sapphire oscillator generating $10-15$-fs pulses ${ }^{13,14}$ with an $\sim 70$-nm bandwidth centered at $800 \mathrm{~nm}$ (KMLabs model TS). The oscillator output is split into pump and probe pulses by a $70 / 30$ beam splitter. The chirped pump pulse duration is set by an SF11 prism pair adjusted to eliminate thirdorder dispersion for the appropriate pulse duration. A collinear pump pulse pair is generated by a Michelson interferometer as a retroreflector.

To optimize the spectral resolution, we consider the effects of pulse shaping on impulsive stimulated Raman scattering. ${ }^{6,9,10}$ In the weak perturbation 


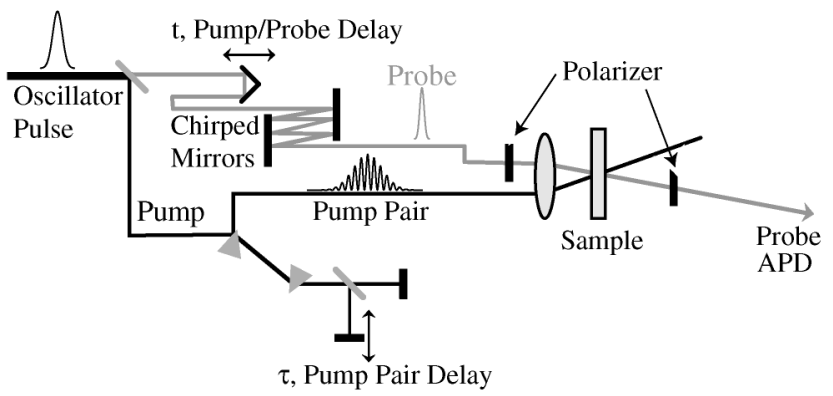

Fig. 1. Experimental setup for mode-selective excitation in liquids: APD, avalanche photodiode.

limit this scattering can be described by a driven damped harmonic oscillator:

$$
\frac{\mathrm{d} Q^{2}}{\mathrm{~d} t^{2}}+2 \gamma \frac{\mathrm{d} Q}{\mathrm{~d} t}+\Omega_{v}{ }^{2} Q=\frac{N}{2}\left(\frac{\mathrm{d} \alpha}{\mathrm{d} Q}\right)_{0}|A(t)|^{2},
$$

where $Q$ is the normalized vibrational distance, $\gamma$ is the coherence decay rate, $\Omega_{v}$ is the vibrational frequency, $N$ is the number density of molecules, $(\mathrm{d} \alpha / \mathrm{d} Q)_{0}$ is the change in polarizability with $Q$, and $|A(t)|^{2} \propto I(t)$ is proportional to the pulse intensity. The amplitude of the excited vibrational mode is given by the magnitude of the Fourier transform of the driving term, or simply by the Fourier transform of $|A(t)|^{2}$, which can be written as $D(\Omega)=$ $\int_{-\infty}^{\infty} \mathrm{d} \omega a_{L}(\omega) a_{L}(\Omega-\omega) \exp \{-i[\phi(\omega)-\phi(\Omega-\omega)]\}$, where $\mathcal{F}\{A(t)\}=a_{L}(\omega) \exp [-i \phi(\omega)]$. The maximum value of this integral at a specific frequency occurs for $\phi(\omega)-\phi(\Omega-\omega)=$ constant. A transform-limited pulse satisifies this condition for every frequency pair within the bandwidth of the laser pulse. A shaped pulse can still excite vibrations over a limited frequency range, with a magnitude bounded by that generated by a transform-limited pulse. From the above analysis it is clear that the resolution of this process is determined by the width of the features in the Fourier transform of $I(t)$ near a desired vibrational frequency.

The intensity profile of the chirped pump pulse pairs used in this experiment can be written in the form $I(t) \propto 1 / 2\left\{a^{2}(t)+r^{2} a^{2}(t-\tau)+2 r a(t) a(t-\tau) \cos \left[\omega_{L} \tau+\right.\right.$ $\phi(t)-\phi(t-\tau)]\}$, where $A(t)=a(t) \exp [-i \phi(t)]$ is the field of a single chirped pump pulse, $\tau$ is the time delay between the pulses, and $r$ is the relative field strength of the pump pulses. In the approximation where $\phi(t)$ is dominated by group-velocity dispersion, the argument of the cosine becomes $\omega_{L} \tau+\pi c_{R}\left[\left(2 \tau t-\tau^{2}\right)\right]$, where $c_{R}$ is the chirp rate $\left[\mathrm{cm}^{-1} / \mathrm{fs}\right]$. Thus the beat frequency of $I(t)$ is given by $c_{R} \tau$, allowing one to adjust the mode excitation frequency by changing the delay. When higher-order spectral phase terms are present, the beat frequency is no longer constant, degrading the resolution. The level of excitation in the impulsive stimulated Raman scattering limit is determined by $D(\Omega)$, which has a dc contribution at zero frequency that is due to the first two terms of $I(t)$, and a sideband at the inverse of the beat period with an envelope given by the Fourier transform of $2 r a(t) a(t-\tau)$.

The resolution (i.e., the spectral width) of the sideband is determined by the Fourier transform of the envelope of the pulse sequence. If we assume a Gaussian pulse shape in time, the sideband magnitude can be written as $|D(\Omega)| \propto \exp \left[-\left(\Omega-\Omega_{0}\right)^{2} / 8 a\right] \exp \left(-a \tau^{2} / 2\right)$, where $a=2 \ln 2 / \tau_{p}{ }^{2}, \Omega_{0}$ is the beat frequency of the chirped pulse pair, and $\tau_{p}$ is the FWHM of $|A(t)|^{2}$. Note that the magnitude of the Fourier transform of a single chirped Gaussian pulse of FWHM $\tau_{p}$ has the same envelope as the chirped pulse pair, but the single-pulse excitation is centered at zero frequency and cannot excite high-frequency modes. Moreover, the magnitude for a single pump pulse is not reduced by the factor $\exp \left(-a \tau^{2} / 2\right)$, which is simply the temporal overlap integral of the delayed pulse pair. This implies that the temporal extent of the interference between the two pump pulses is equal to the duration of a single pump pulse and, as a result, that the resolution is independent of the pump pulse delay. Furthermore, the resolution is inversely proportional to the duration of the chirped pump pulse. Thus the optimal pump pulse duration is the inverse of the linewidth of the Raman mode.

To optimize the resolution we use the fact that the third-order dispersion of a prism pair is the opposite to that of the material. Thus an optical configuration can be set up that cancels the third-order dispersion while imparting a linear chirp. ${ }^{13,15}$ In practice, first we calculate the insertion and separation that give the desired chirped pulse duration. Based on this calculation, we set the SF11 prism separation. Then, we measure the cross correlation between the pump pulse pair and the transform-limited probe pulse, which provides a direct measure of $I(t)$ for the pump pulse pair. We minimize the width of the Fourier transform of the cross-correlation signal, which determines the excitation resolution, by adjusting the glass insertion to minimize the third-order dispersion. Once the resolution of the pump pulse is optimized, we adjust the time delay to tune the excitation frequency. We can determine the average chirp rate of the pulse pair by taking the ratio $\Delta v / \Delta t$, where $\Delta v$ is the spacing between the modulations of the pump pulse pair spectrum and $\Delta t$ is the spacing between modulation of $I(t)$ measured by the cross correlation. Once the chirp rate is determined, the resonant frequency can be measured simply by measuring the pulse spectrum.

Figure 2(a) shows the measured transient birefringence response of the $216-\mathrm{cm}^{-1}\left(v_{2}\right)$ and $314-\mathrm{cm}^{-1}\left(v_{4}\right)$ modes in the $\mathrm{CCl}_{4}$ as a function of the time delay between the pump and the probe. The nuclear response of the system is most easily observed as residual response at late times. The amplitude of this residual response is correlated to the beat frequency of the pump pulse pair. Data from shorter time delays show both the electronic Kerr effect and the nuclear motion. A Fourier transform of the leading edge of the signal results in a frequency spike at the beat frequency of the pump pulse pair, whereas the Fourier transform of the tail of the signal [see Fig. 2(b)] labels the frequency of the excited mode. Here, the duration of the chirped pump pulse was set to $750 \mathrm{fs}$ (implying a $1.276-\mathrm{cm}^{-1} / \mathrm{fs}$ chirp rate) at a prism separation of $5.5 \mathrm{~cm}$ and an insertion of $2.5 \mathrm{~cm}$, yielding a resolution of $26 \mathrm{~cm}^{-1}$, which compares well with the Raman 


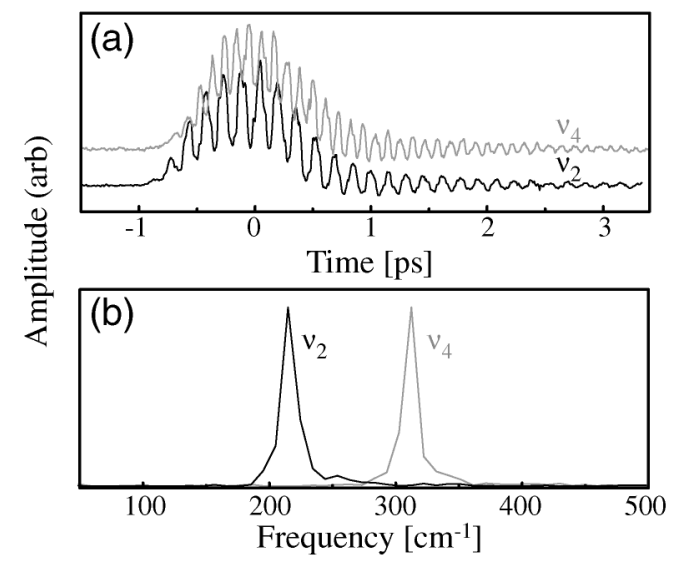

Fig. 2. Kerr effect signals in $\mathrm{CCl}_{4}$, as a function of pump-probe delay, for two pump pulse sequences that preferentially excite the $v_{2}$ or $v_{4}$ modes.

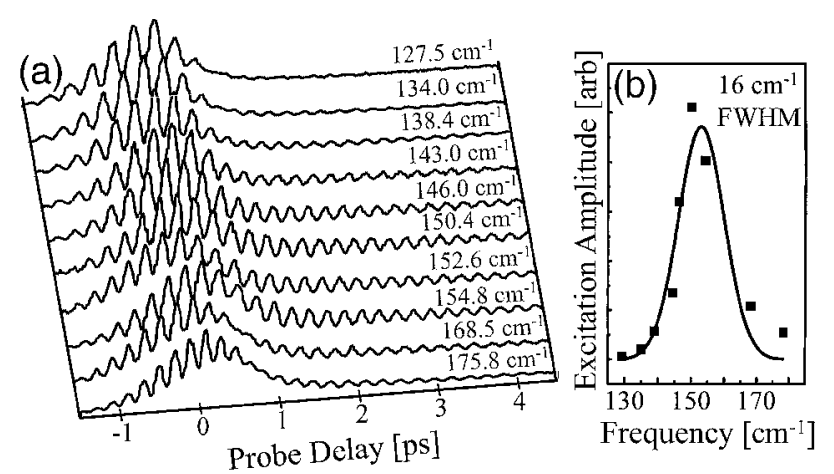

Fig. 3. Kerr effect signal in $\mathrm{CHBr}_{3}$, as a function of pump-probe delay. The curves represent different time delays between the two pulses that were used to generate the pump pulse sequence, and hence different excitation pulse sequences. Inset, experimentally measured resolution.

transition linewidth of $8.2 \mathrm{~cm}^{-1}$. Generally, because the dispersion of both the prism and the material scales linearly, the optimum setup has a fixed ratio of prism separation to material insertion of 2.22 .

We repeated the above experiment with $\mathrm{CHBr}_{3}$, which has a narrower linewidth, $4.5 \mathrm{~cm}^{-1}$. Figure 3 shows the measured transient birefringence of the 154- $\mathrm{cm}^{-1}$ mode in $\mathrm{CHBr}_{3}$ as a function of the pump and probe time delay. The inset of Fig. 3 shows the amplitude of the Fourier transform of the nuclear tail as the driving frequency is tuned. This inset shows a FWHM resolution of $15 \mathrm{~cm}^{-1}$ (or $\sim 80$ times better than transform-limited excitation) for a $1500-\mathrm{fs}$ chirped pump pulse (prism separation, $12 \mathrm{~cm}$; insertion, $5.4 \mathrm{~cm}$ ). This resolution compares favorably with the ratio of the chirped to the initial pulse duration, which for $\mathrm{CHBr}_{3}$ is $\sim 1500 / 16$, or 94 .

Because one accomplishes tuning by changing the time delay, this technique is amenable to rapid tuning and lock-in detection. For example, a major issue in CARS microscopy is to distinguish the nonres- onant electronic response from the resonant nuclear response of the target species. By dithering the time delay between the two pulses and using lock-in detection (at the dither frequency or higher harmonics) one can directly extract the resonant response in cases such as single-pulse CARS for which the probe signal is not sensitive to the phase of the material response. This can be done with a single laser or a pair of separate time-synchronized lasers. ${ }^{16}$

In conclusion, we have demonstrated that, by using a single, femtosecond laser and simple optics, we can selectively excite vibrational modes in $\mathrm{CCl}_{4}$ and $\mathrm{CHBr}_{3}$ liquids with very high spectral resolution. The resolution is more than 80 times higher than the laser bandwidth. Our setup represents a significant improvement in spectral resolution over previous designs, as well as a simplification in setup.

The authors gratefully acknowledge support from the National Science Foundation. H. C. Kapteyn's e-mail address is kapteyn@jila.colorado.edu.

*Permanent address, Eugene F. Merkert Chemistry Center, Boston College, Chestnut Hill, Massachusetts 02467.

\section{References}

1. B. Schrader, Infrared and Raman Spectroscopy (VCH, Weinheim, Germany, 1995).

2. M. D. Levenstein, Introduction to Nonlinear Laser Spectroscopy (Academic, San Diego, Calif., 1982).

3. M. D. Fayer, Ultrafast Infrared and Raman Spectroscopy (Marcel Dekker, New York, 2001).

4. A. Zumbusch, G. R. Holtom, and X. S. Xie, Phys. Rev. Lett. 82, 4142 (1999).

5. S. De Silvestri, J. G. Fujimoto, E. P. Ippen, E. B. Gamble, L. R. Williams, and K. A. Nelson, Chem. Phys. Lett. 116, 146 (1985).

6. A. M. Weiner, D. E. Leaird, G. P. Wiederrecht, and K. A. Nelson, Science 247, 1317 (1990).

7. D. Oron, N. Dudovich, D. Yelin, and Y. Silberberg, Phys. Rev. Lett. 88, 063004 (2002).

8. L. Dhar, J. A. Rogers, and K. A. Nelson, Chem. Rev. 94, 157 (1994).

9. T. C. Weinacht, R. Bartels, S. Backus, P. H. Bucksbaum, B. Pearson, J. M. Geremia, H. Rabitz, H. C. Kapteyn, and M. M. Murnane, Chem. Phys. Lett. 344, 333 (2001).

10. R. A. Bartels, T. C. Weinacht, S. R. Leone, H. C. Kapteyn, and M. M. Murnane, Phys. Rev. Lett. 88, 033001 (2002).

11. A. S. Weling, Appl. Phys. Lett. 64, 137 (1994).

12. N. Dudovich, D. Oron, and Y. Silberberg, Nature 416, 512 (2002).

13. M. T. Asaki, C. P. Huang, D. Garvey, J. Zhou, H. C. Kapteyn, and M. M. Murnane, Opt. Lett. 18, 977 (1993).

14. J. P. Zhou, G. Taft, C. P. Huang, M. M. Murnane, H. C. Kapteyn, and I. P. Christov, Opt. Lett. 19, 1149 (1994).

15. C. P. Huang, H. C. Kapteyn, J. W. McIntosh, and M. M. Murnane, Opt. Lett. 17, 139 (1992).

16. R. K. Shelton, S. M. Foreman, L. S. Ma, J. L. Hall, H. C. Kapteyn, M. M. Murnane, M. Notcutt, and J. Ye, Opt. Lett. 27, 313 (2002). 